\title{
Study on Film Characteristics of Piston-Cylinder Interface of High Pressure Common Rail Radial Piston Pump with Viscosity- Temperature-Pressure Effect
}

\author{
Bo Qi, Yong Zhang, Guoyou Meng, and Yao Ding \\ School of mechanical and automotive engineering, South China University of Technology, China
}

\begin{abstract}
Aiming at the inaccuracy of equivalent viscosity method in solving the film characteristics of piston-cylinder interface of high-pressure common rail radial piston pump, the film characteristics equation of piston-cylinder interface was established based on the theory of thermo-hydrodynamic lubrication. Through the solution, the thermal properties of the piston-cylinder interface film, which accounted for viscosity-temperature-pressure effect, were studied. The effects of cam speed and film inlet pressure on the characteristics of the piston-cylinder interface film were discussed. The conclusion has certain theoretical and engineering application value for the design and basic research of piston-cylinder interface.
\end{abstract}

\section{Introduction}

In recent years, with the increasingly stringent emission regulations and the continuous progress of diesel engine electronic control technology, high-pressure common rail fuel injection system with its remarkable advantages has become the main trend of modern diesel engine technology development. Piston-cylinder is the key friction pair of piston pump of the common rail system. Under the influence of lubricating film pressuretemperature-viscosity, the lubricating state changes constantly.

When the influence of temperature and pressure on film viscosity has been considering, the equivalent viscosity method is generally used to solve it. In this method, the film viscosity is calculated according to the heat balance of the piston-cylinder or the average value of the pressure and temperature of the inlet and outlet film. This method can simply analyse the film characteristics of piston-cylinder interface, but the results are not accurate. The distribution of the film pressure temperature and viscosity of the piston-cylinder interface, especially the maximum temperature and pressure position, are critical for the design of the pistoncylinder. In order to ensure the reliability and durability of the common rail system, it is necessary to study the film characteristics of piston-cylinder interface of the high-pressure common rail radial piston pump, including viscosity-temperature-pressure effect.

\section{Mathematical model}

The structural model of the piston-cylinder interface is shown as Fig.1. The centre of the end face of the cylinder is taken as the origin, e as the eccentric distance of the piston-cylinder interface and $\theta$ as the eccentric angle of the piston. A is near the piston cavity and B is near the slipper. The film of piston-cylinder interface is expanded along the circumference direction, as shown in Fig.2. $x$ is the direction of the cylinder circumference, $y$ is the axial direction of the cylinder, and $z$ is the direction of the piston-cylinder interface film thickness. $u, v$ and $w$ are the speed components of lubricating film in $x, y$ and $z$ directions.

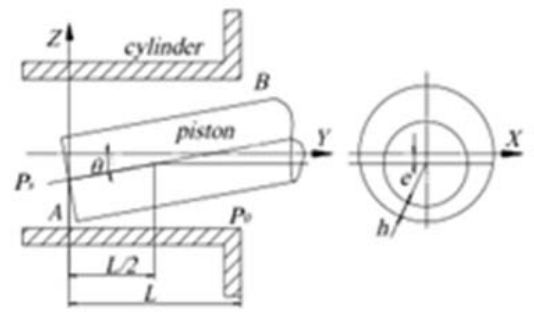

Fig. 1. Eccentric model of piston-cylinder interface.

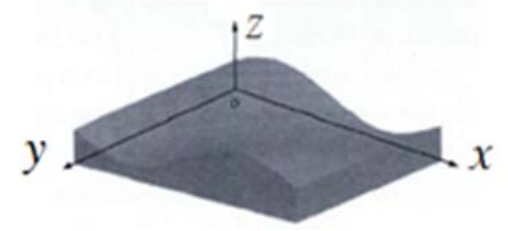

Fig. 2. Lubricant film model for piston-cylinder interface.

\subsection{Reynolds equation of lubrication film}

The inner film of piston-cylinder interface is annular gap flow, which belongs to laminar flow. Under the viscosity-temperature-pressure effect, the Reynolds 
equation [1] of the piston-cylinder interface lubricant film can be expressed as:

$$
\frac{\partial}{\partial x}\left(\frac{\rho h^{3}}{\mu} \frac{\partial p}{\partial x}\right)+\frac{\partial}{\partial y}\left(\frac{\rho h^{3}}{\mu} \frac{\partial p}{\partial y}\right)=6 V_{1} \frac{\partial(\rho h)}{\partial y}+12 \frac{\partial(\rho h)}{\partial t}
$$

\subsection{Energy equation of lubrication film}

The energy equation the piston-cylinder interface film can be expressed as [2-6]:

$$
\frac{\partial}{\partial x}\left(\frac{\rho h^{3}}{\mu} \frac{\partial p}{\partial x}\right)+\frac{\partial}{\partial y}\left(\frac{\rho h^{3}}{\mu} \frac{\partial p}{\partial y}\right)=6 V_{1} \frac{\partial(\rho h)}{\partial y}+12 \frac{\partial(\rho h)}{\partial t}
$$

where $\rho$ is the lubricating film density of pistoncylinder interface, $c$ is specific heat at constant pressure $k$ is the thermal conductivity, $\mu$ is oil viscosity.

\subsection{Equation of thickness of lubricating film}

The equation of film thickness after film expansion is shown as follows:

$$
h(\theta, y)=C+[e+\theta(y-0.5)] \cos (\varphi)
$$

where $C$ is the average clearance between piston and cylinder, $\varphi$ is the angle between the central line of any two sections along the piston axis and the x-positive semi-coordinate axis.

\subsection{Equation of viscosity-temperature-pressure of piston-cylinder interface film}

There is no uniform method to calculate the viscositytemperature-pressure relation of lubricating film [7-9]. Roelands formula can describe accurately the relationship among viscosity, temperature and pressure, as shown in the following formula:

$$
\mu=\mu_{0} \exp \left\{\left(\operatorname{In} \mu_{0}+9.67\right)\left[-1+\left(1+5.1 \times 10^{-9} P\right)^{Z}\left(\frac{T-138}{T_{0}-138}\right)^{-S}\right]\right\}
$$

in the equation above,

$$
Z=\alpha /\left[5.1 \times 10^{-9}\left(\operatorname{In} \mu_{0}+9.67\right)\right] ; \quad S=\beta\left(T_{0}-138\right) /\left(\operatorname{In} \mu_{0}+9.67\right)
$$

\subsection{Heat conduction equation of piston-cylinder interface}

The heat conduction equation of piston-cylinder temperature field can be expressed as [10]:

$$
\begin{aligned}
& c_{c} \rho_{c} \frac{\partial T_{c}}{\partial t}=k_{c}\left(\frac{\partial^{2} T_{c}}{\partial r_{c}^{2}}+\frac{1}{r_{c}} \frac{\partial T_{c}}{\partial r_{c}}+\frac{1}{r_{c}^{2}} \frac{\partial^{2} T_{c}}{\partial \theta^{2}}+\frac{\partial^{2} T_{c}}{\partial y_{c}{ }^{2}}\right) \\
& c_{p} \rho_{p} \frac{\partial T_{p}}{\partial t}=k_{p}\left(\frac{\partial^{2} T_{p}}{\partial r_{p}{ }^{2}}+\frac{1}{r_{c}} \frac{\partial T_{p}}{\partial r_{p}}+\frac{1}{r_{p}{ }^{2}} \frac{\partial^{2} T_{p}}{\partial \theta^{2}}+\frac{\partial^{2} T_{p}}{\partial y_{p}{ }^{2}}\right)
\end{aligned}
$$

where $c_{\mathrm{c}} 、 \rho_{\mathrm{c}} 、 k_{\mathrm{c}} 、 c_{\mathrm{p}}, \rho_{\mathrm{p}}, k_{\mathrm{p}}$ is the specific heat, density and heat conduction coefficient of cylinder and piston. $T_{\mathrm{c}}, T_{\mathrm{p}}$ is the cylinder and piston temperature. $r_{\mathrm{c}}, y_{\mathrm{c}}$, $y_{\mathrm{p}}, r_{\mathrm{p}}$ is the axial and radial coordinates of cylinder and piston; $R \mathrm{c} 1$ and $R_{\mathrm{c} 2}$ are the inner and outer diameter of cylinder.

\section{Simulation results and analysis}

The generalized Reynolds equation of piston-cylinder interface is an elliptic equation. The Reynolds boundary and explicit point iteration method have been used to solve the equation. The numerical solution has been carried out in MATLAB software [11]. Cam speed and film inlet pressure are the main parameters that affect the film characteristics of piston-cylinder interface. When the working condition is fixed, the greater the cam pressure angle, the greater the external load the piston bears, the worst film characteristics of the pistoncylinder interface. The influence of cam speed and film inlet pressure on the film characteristics of pistoncylinder interface with the effect of viscositytemperature-pressure has been studied when the cam pressure angle is the maximum.

\subsection{Effect of cam speed on lubrication characteristics of piston-cylinder interface}

Taking the inlet pressure of the piston-cylinder interface as $160 \mathrm{MPa}$, the temperature of $30^{\circ} \mathrm{C}$ and the cam speed of $2500 \mathrm{r} / \mathrm{min}$ for example, the distribution of the pressure, temperature and viscosity of the lubricating film of the piston-cylinder interface are shown respectively in Fig.3.

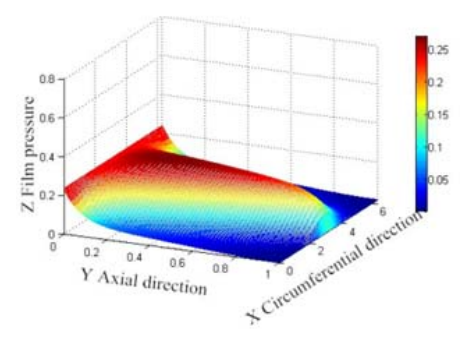

a. Film pressure distribution

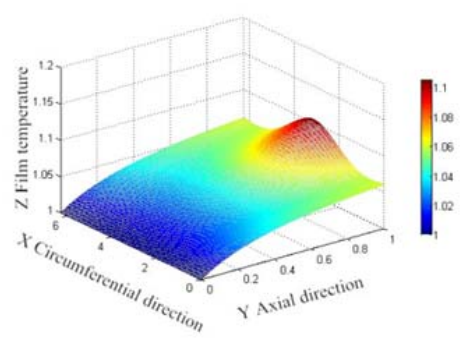

b. Film temperature distribution

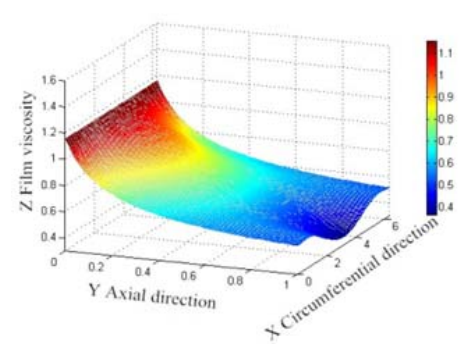

c. Film viscosity distribution

Fig. 3. Characteristic distribution of piston-cylinder interface lubricant film. 
The pressure of film changes obviously near the film inlet and outlet, and the extreme point appears near the film inlet. In the same axial position, the smaller the film thickness, the greater the pressure. The film temperature rises gradually from the inlet to the outlet and reaches the maximum value at the outlet. Under the interaction of pressure and temperature, the film viscosity changes continuously and reaches the minimum value at the outlet.

Under different cam speeds, the film pressure changes along the axis direction corresponding to the unfolding angle $\pi$, as shown in Fig.4.

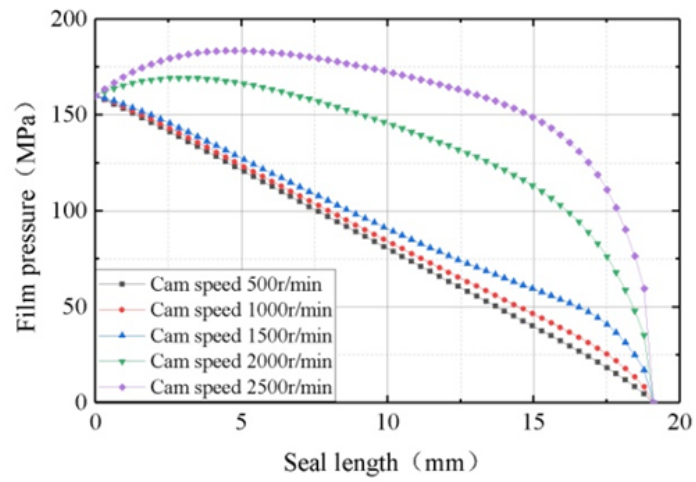

Fig. 4. The variation of the film pressure along the axis of the expansion angle under different cam speeds.

When the rotating speed is small, the film pressure decreases linearly along the axial direction. When the cam speed increases to a certain value, the film pressure changes linearly near the film inlet and non-linearly near the outlet. When the cam speed is greater than 2000 $\mathrm{r} / \mathrm{min}$, the film pressure first increases and then decreases, and the pressure changes significantly near the outlet. The larger the cam speed becomes, the greater the film pressure changes inside the piston-cylinder interface.

Under different cam speeds conditions, the film temperature changes along the axis direction corresponding to the unfolding angle $\pi$, as shown in Fig.5.

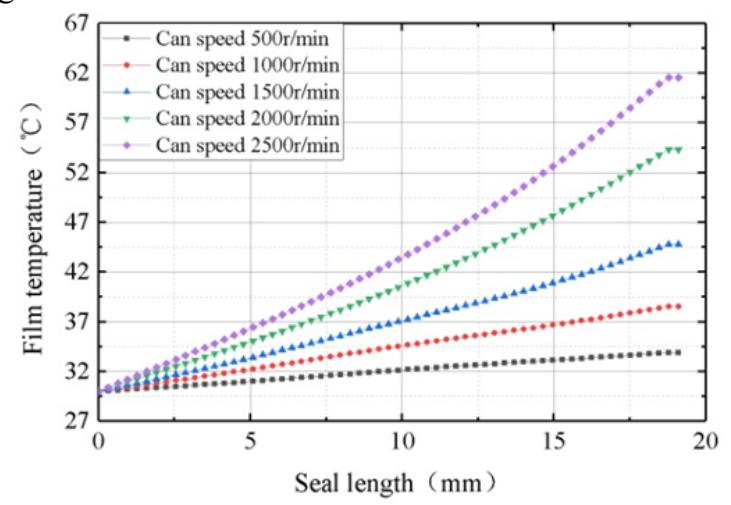

Fig. 5. The variation of film temperature along the axis of the expansion angle under different cam speeds.

Under the different rotational speed conditions, the film temperature of the piston-cylinder interface increases along the corresponding axial direction of the unfolding angle $\pi$, and the greater the rotational speed, the greater the temperature. When the rotational speed is low, the film temperature changes linearly along the axial direction. When the rotational speed is high, the change rate of the film temperature along the axial direction increases gradually.

Under different cam speed conditions, the film viscosity changes along the axis direction corresponding to the unfolding angle $\pi$, as shown in Fig. 6.

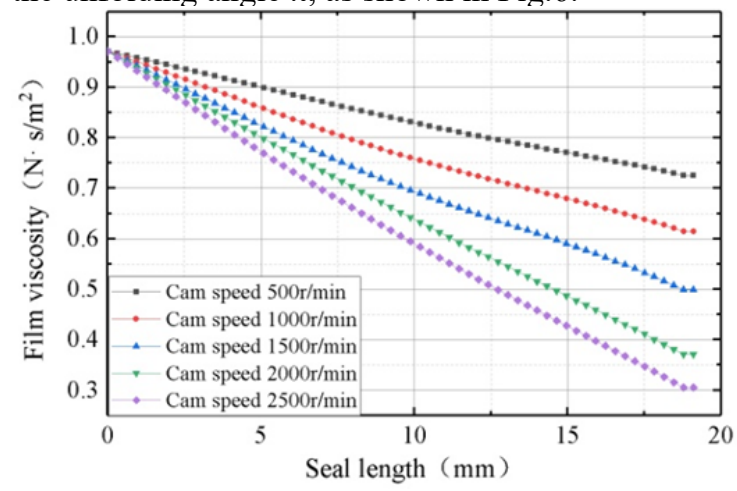

Fig. 6. The variation of film viscosity along the axis of the expansion angle $\pi$ at different cam speeds.

The film pressure of the piston-cylinder interface decreases gradually along the direction of the corresponding axis of the unfolding angle $\pi$, and the film temperature increases gradually along the direction of the corresponding axis of the unfolding angle $\pi$. According to the viscosity-temperatur-pressure effect formula, the lower the film pressure and the higher the temperature, the lower the film viscosity. And the film viscosity decreases along the axial direction under the interaction of film pressure and temperature.

\subsection{Influence of film inlet pressure on film characteristics of piston-cylinder interface}

Taking the inlet pressure of the piston-cylinder interface as $120 \mathrm{MPa}$, the temperature as $30^{\circ} \mathrm{Cand}$ the cam speed as $2500 \mathrm{r} / \mathrm{min}$ for example, the distributions of the pressure, temperature and viscosity of the lubricating film of the piston-cylinder interface are shown respectively as Fig. 7.

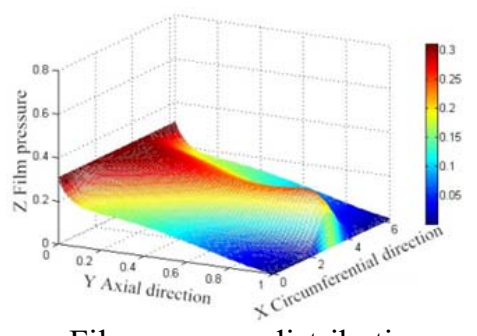

a Film pressure distribution 


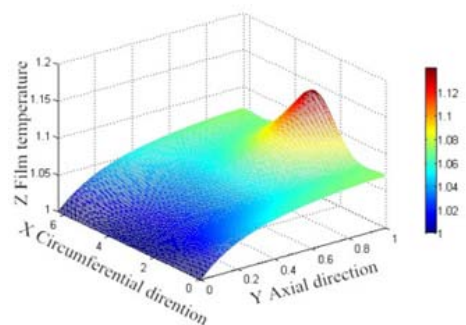

b Film temperature distribution

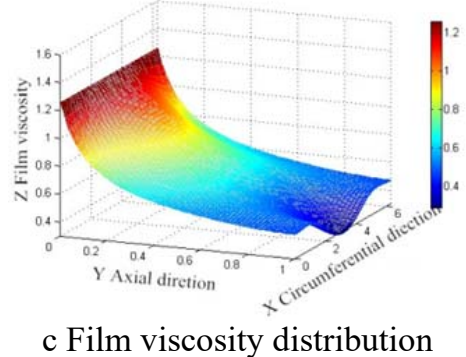

Fig. 7. Characteristic distribution of piston-cylinder interface lubricant film.

The pressure and temperature distribution of lubricating film are closely related to the film thickness of piston-cylinder interface. At the same axial position, the greater the film thickness, the higher the pressure and temperature. According to the viscosity-temperaturepressure formula, the viscosity of film is in direct proportion to the e power of film pressure, and is inverse proportion to the e power of temperature. Under the influence of film pressure and temperature, the viscosity of film changes obviously.

Under different pressure inlet conditions, the change of film pressure along the axial direction corresponding to the unfolding angle $\pi$ is shown in Fig.8.

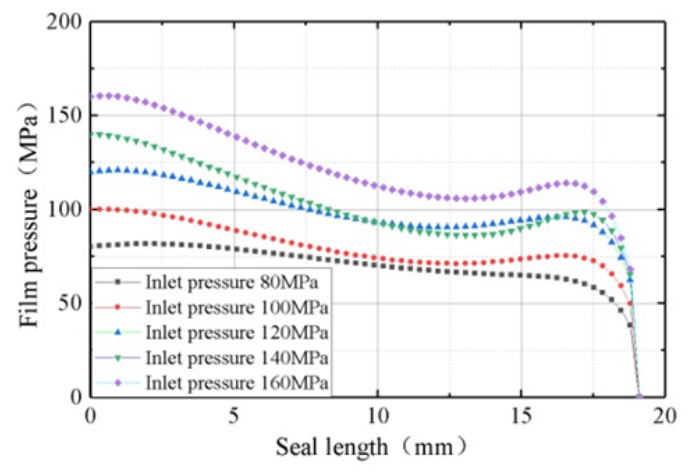

Fig. 8. The variation of the film pressure along the axis of the expansion angle $\pi$ under different inlet pressures.

When the film inlet pressure is low, the film pressure of the piston-cylinder interface decreases gradually along the axial direction corresponding to the unfolding angle $\pi$. When the inlet pressure is large, the film pressure decreases first and then increases along the axial direction. The film pressure is located at the inlet and the extreme point near the outlet.

Under different pressure inlet conditions, the change of film temperature along the axial direction corresponding to the unfolding angle $\pi$ is shown in Fig.9.

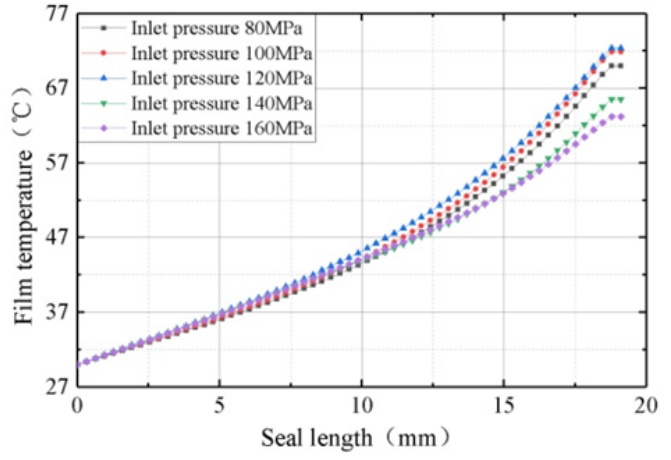

Fig. 9. The variation of film temperature along the axis of the expansion angle $\pi$ under different inlet pressures.

The film temperature increases gradually along the axial direction and reaches the maximum value at the outlet of the film. The film temperature varies little at the inlet of different temperatures if the sealing position is within the range $0 \mathrm{~mm}$ to $10 \mathrm{~mm}$. As the increase of sealing position, the temperature difference becomes obvious gradually. According to the energy equation of piston-cylinder interface, the film heat source is composed of differential pressure flow and shear flow. With the increase of film pressure, the eccentricity angle and offset of piston gradually decrease, and the interaction between them makes the temperature change, caused by different inlet pressure, more complex.

Under different inlet pressure, the film viscosity changes along the axial direction corresponding to the unfolding angle $\pi$ is shown in Fig. 10 .

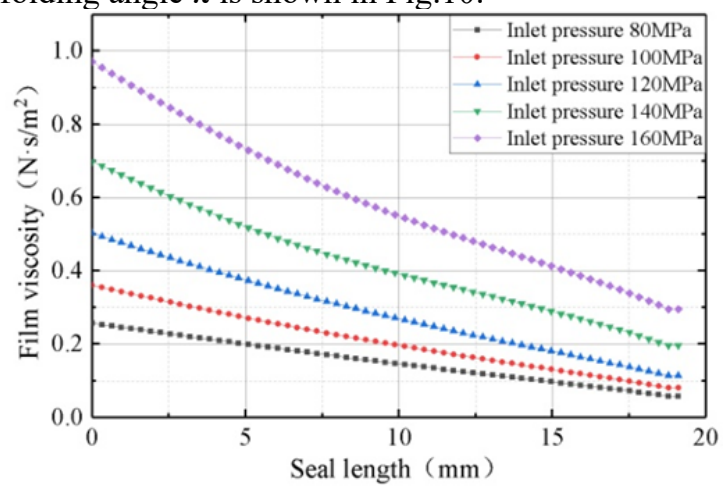

Fig. 10. Film viscosity variation along the spread angle $\pi$ corresponds to axial under different inlet pressures.

The viscosity of film decreases along the axis corresponding to the unfolding angle $\pi$, and the greater the inlet pressure, the more obvious the viscosity change. At the inlet of the film, the pressure is the main factor that affects the viscosity of the film, and the temperature at the exit is the main factor that affects the viscosity. The higher the inlet pressure, the smaller the secondary heat of the piston. Because of the interaction influence of film pressure and temperature, the viscosity changes linearly at low pressure, and non-linearly at high pressure. 


\section{Conclusion}

Based on the theory of thermo-hydrodynamic lubrication, a thermo-hydrodynamic lubrication model of pistoncylinder interface of high-pressure common rail radial piston pump has been established in this paper. By solving Reynolds equation, film thickness equation, energy equation and heat conduction equation, the film characteristics of piston-cylinder interface under different working conditions have been discussed. The following important conclusions can be drawn from the study.

(1) Under the fixed inlet pressure and temperature of the film, the film pressure decreases linearly along the axial direction when the rotational speed is low. When the rotational speed is high, the film pressure increases first, then decreases along the axial direction, and changes sharply near the outlet. The film temperature increases along the axis corresponding to the film unfolding angle $\pi$ and reaches its maximum near the film outlet. When the rotating speed is low, the film temperature increases first and then decreases along the direction of the expansion line. When the rotating speed is high, the film temperature increases gradually, and the temperature change rate increases gradually. If the film pressure becomes lower and the temperature becomes, also, higher, the viscosity of the film will be lower. The viscosity of film decreases continuously along the axial direction under the interaction of film pressure and temperature.

(2) Under the fixed cam speed and inlet temperature, the film pressure decreases gradually from the inlet to the outlet when the inlet pressure is low. When the film pressure is high, the film pressure will reach an extreme value near the outlet. As the increase of seal length, the film pressure first decreases and then increases. Under different inlet pressure, the temperature gradually increases along the axis and reaches the maximum near the outlet. Under different inlet pressure, the film viscosity decreases along the axis, and reaches the minimum value on the axis corresponding to the unfolding angle $\pi$.

\section{References}

1. Schlichting H. Boundary-layer theory. New York: Mc Graw-Hill.(1979)

2. Caika C, Bukovnik S, Offner G et al. Elastichydrodynamic lubrication model with pressure, temperature and shear rate dependent [C]. International Conference on Tribology.(2006)

3. Uwe wieczorek, Monika Ivantysynova. Computer aided optimization of bearing and sealing and sealing gap in hydrostatic machines the simulation tool CASPAR [J]. International Journal of Fluid Power.3(1):7-20 (2002)

4. Ivantysynova, M. Temperaturfeld im schmierspalt zwischen kolben und zylinder einer axialkolbenmaschine. Maschinenbautechnik. 34:532535(1985)

5. Walowit J A, Anno J N. Modern developments in lubrication mechanics. London: Applied Science Publishers Ltd. (1975)

6. Liu X L, Yang P R.Analysis of non- newtonian thermal elastohydynamic lubrication based on surface dimple phenomenon[J].Tribology. 23(5): 406-410 (2003)

7. Meng Q R,Hou Y F.Numerical simulation on transient behavior of hudro-viscous drive speed regulating start [J]. Tribology. 29(9):418-424 (2009)

8. Zhang J J,He Z X,Wang L. Influence of viscositypressure coefficient on non-steady-state thermal elastohydrodynamic lubrication[J]. Coal Mine Machinery. 27(12):70-72 ( 2006)

9. Lu F X,Long S Y. Thermal elastohdrodynamic lubrication analysis of point contact for aviation transmission[J]. Journal of Mechanical. 39(6):9-15 (2015)

10. Incropera $\mathrm{F} P$, De Witt $\mathrm{D} P$, Bergman $\mathrm{T} \mathrm{L}$. Fundamentals of heat and mass transfer. 6th ed. New York: John Wiley \& Sons.(2007)

11. Wang Z S,Mu G H,Niu C J. Reserch on simulation of oil film charateristics of dynamic pressure bearing based on matlab $[\mathrm{J}]$. Design Research.40(2):9-11 (2013) 